Check for updates

Cite this: Mater. Chem. Front. 2020, 4, 1706

Received 24th February 2020 Accepted 6th April 2020

DOI: 10.1039/d0qm00098a

rsc.li/frontiers-materials

\title{
Each phenyl group performs its own functions on luminescence: phenyl substituted effect in tetraphenylpyrazine $\dagger$
}

\author{
Haozhong Wu, ${ }^{a}$ Xiaojuan Song, ${ }^{b}$ Bing Zhang, ${ }^{a}$ Zhiming Wang, (D) *a Tian Zhang, (D) *b \\ Anjun Qin (D) and Ben Zhong Tang (iD ac
}

\begin{abstract}
Aggregation-induced emission (AIE) has drawn considerable attention owing to its interesting phenomenon, and the AIE mechanisms of different molecule systems have been gradually revealed. Here, we investigated the difference in 3-carbazole-pyrazine-based isomers and explored the effects of three substituted phenyl groups (ortho, meta and para) on the tetraphenylpyrazine derivatives (TPPs). The meta- and para-phenyl groups could slightly and significantly adjust their emission properties, respectively. The ortho-phenyl group could distort the pyrazine plane owing to the large steric hinderance of the two moieties; this triggered many molecular motions and led to the wastage of excited state energy, stimulating the AIE characteristics of TPPs. This result is demonstrated for other pyrazine derivatives and can serve as a design strategy of AIE molecules.
\end{abstract}

\section{Introduction}

Organic $\pi$-conjugated fluorescent materials have been investigated in the past several decades owing to their promising applications in organic light-emitting diodes (OLEDs), ${ }^{1-6}$ chemical sensors ${ }^{7-11}$ and biological imaging. ${ }^{12-17}$ However, the notorious problem of these classical luminescent materials is the familiar aggregation-caused quenching (ACQ), i.e., they weakly emit in their aggregated states such as powder and crystals compared to their dilute solutions. ${ }^{18}$ To overcome this issue, Tang et al. proposed the opposite phenomenon, namely, aggregation-induced emission (AIE). From then on, many AIE luminogens (AIEgens) have been explored and developed in multiple research fields. ${ }^{19-22}$

Compared to common fluorophores, AIEgens undergo some complicated relaxation paths in their excited states; therefore, several mechanisms, such as restricted intramolecular motions

\footnotetext{
${ }^{a}$ State Key Laboratory of Luminescent Materials and Devices, Center for Aggregation-Induced Emission, Key Laboratory of Luminescence from Molecular Aggregates of Guangdong Province, Guangzhou International Campus, South China University of Technology (SCUT), Guangzhou 510640, China. E-mail: wangzhiming@scut.edu.cn

${ }^{b}$ School of Chemistry and Chemical Engineering, Shandong University of Technology, Zibo 255049, P. R. China. E-mail: tzhang@sdut.edu.cn

${ }^{c}$ Department of Chemistry, The Hong Kong University of Science \& Technology (HKUST), Clear Water Bay, Kowloon, Hong Kong, China. E-mail: tangbenz@ust.hk $\dagger$ Electronic supplementary information (ESI) available: Synthesis, characterization, TGA curves, DSC curves, CV curves PL spectra. CCDC 1976103 and 1976104. For ESI and crystallographic data in CIF or other electronic format see DOI: 10.1039/ d0qm00098a
}

(RIMs) and that of J-aggregates, have been put forward to explain the AIE phenomenon. Among them, RIM has been widely acknowledged as one of the origins of AIE. ${ }^{19,20,23-26}$ From single molecular states to aggregated states, the restricted moieties of different molecular systems are not identical (Fig. 1A-C). In dilute solutions, twisting double-bond of tetraphenylethene (TPE) and its derivatives can lead the potential energy surface to the conical intersection and return to the ground states without strong fluorescence. ${ }^{27}$ For the TICT model compounds, the nearly perpendicular donor-acceptor structures hinder the radiative decay process because of less orbital overlap and accelerate internal crossing due to the small energy gaps. ${ }^{28-31}$ The aromaticity reversal of cyclooctatetrathiophene produces many structural vibrations; ${ }^{32}$ therefore, weak emissions can be observed in these molecules. Once aggregated, the restriction of all the abovementioned motions leads to bright fluorescence.

Tetraphenylpyrazine (TPP) has been discovered as an AIE unit; it is often considered that the obstruction of the rotation with respect to the peripheral phenyl groups leads to AIE features (Fig. 1D). ${ }^{33}$ Lately, the X-ray diffraction of the crystalline structure and theoretical calculations have demonstrated that the pyrazine cores of TPP-based molecules have some distortion, which can create a number of vibrational motions in their excited states. ${ }^{34}$ Furthermore, the conjugation along the substituted axis mostly determines the transition process for single substituted compounds and the off-axis phenyl groups can influence this slightly. ${ }^{35}$ Therefore, it is important to investigate the role of each phenyl group on TPP for the 


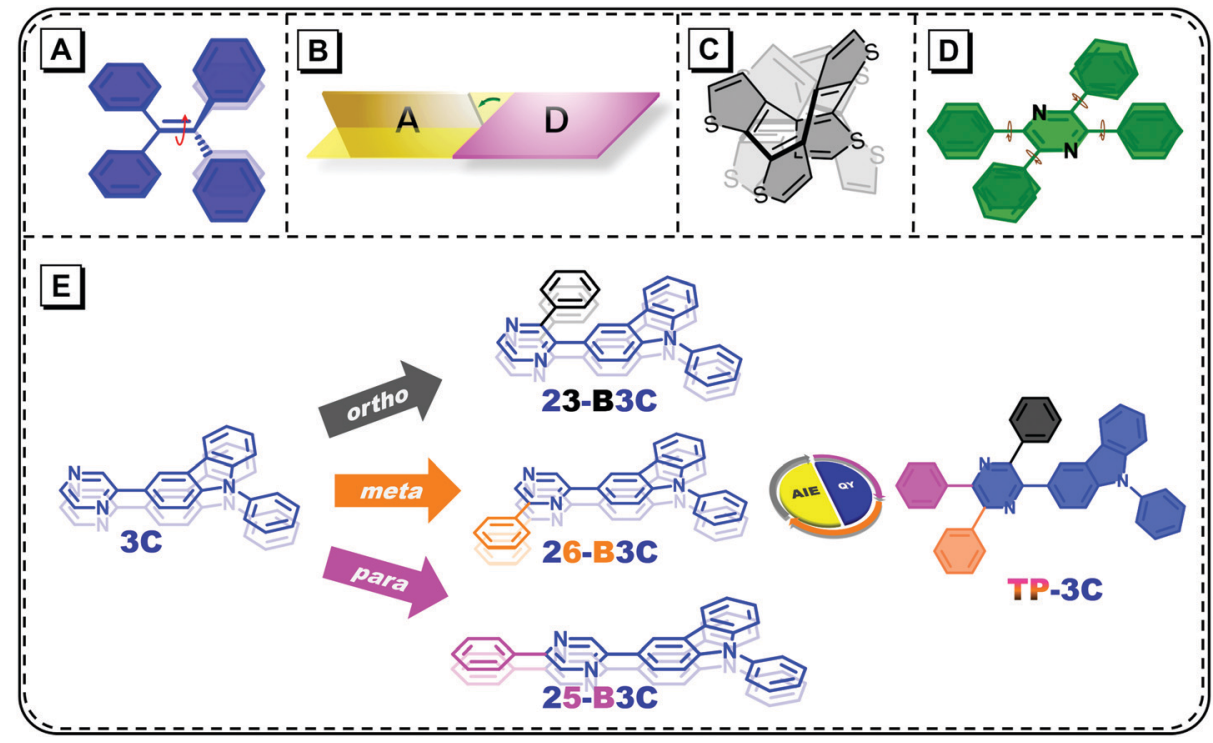

Fig. 1 The distortion modes of (A) TPE, (B) TICT molecules, (C) cyclooctatetrathiophene and (D) TPP; (E) the chemical structures of five pyrazinecarbazole derivatives.

further molecular design and modification. In this work, 3-carbazole was chosen as the chromophore owing to its rigidity and weak electron-donating ability. The phenyl group was introduced into the ortho, meta and para positions of pyrazine and three compounds, namely, 23-B3C, 26-B3C and 25-B3C were obtained, respectively. Also, a 3-carbazole-substituted compound $3 \mathrm{C}$ and a fully substituted compound TP-3C were synthesized for comparison (Fig. 1E).

\section{Results and discussion}

\section{Synthesis and single crystal structure}

The synthetic route of the five target products is outlined in Fig. S1 (ESI $\dagger$ ). The details of the synthesis process and characterization are provided in the ESI $\dagger$ (Fig. S2-S15). 3C was prepared by a Suzuki coupling reaction with (9-phenyl-9Hcarbazol-3-yl)boronic acid and 2-bromopyrazine. 23-B3C, 25-B3C and 26-B3C were also obtained from the corresponding dibromopyrazine, (9-phenyl-9H-carbazol-3-yl)boronic acid and phenylboronic acid via a Suzuki coupling reaction. TP-3C was obtained using 3-bromo-9-phenylcarbazole and was synthesized via the Sonogashira coupling reaction, oxidation reaction and Schiff base cycling reaction successively. ${ }^{34}$ These final compounds were soluble in common organic solvents, such as tetrahydrofuran (THF) and dichloromethane, but not in methanol and water.

To investigate the substituted distinction on crystalline packing, we tried to prepare single crystals, but only 23-B3C (CCDC 1976103 $\dagger$ ) and 25-B3C (CCDC 1976104 $\dagger$ ) were obtained by evaporation from methanol/dichloromethane or methanol/ THF mixtures. The detailed analysis of the resulting X-ray diffraction (XRD) data is shown in the ESI. $\dagger$ As illustrated in Fig. 2A, 23-B3C adopts a twisted conformation with a distortion of approximately $44^{\circ}$ between pyrazine and 3-carbazole due to the steric hindrance between 3-carbazole and the phenyl group, which can prohibit the notorious $\pi-\pi$ stacking in a manner similar to that of many classic AIEgens such as TPE and polyphenylsilole (Fig. 2A and E). ${ }^{36}$ Some $\mathrm{C}-\mathrm{H} \cdots \pi$ intermolecular interactions are beneficial to restrict molecular motions (Fig. 2B). Hence, we supposed that 23-B3C had the AIE characteristic based on this molecular structure and packing pattern. Nevertheless, the large pyrazine-(3-carbazole) torsion angle indicated that weak fluorescence may be observed in its solid state. In the 25-B3C crystal, the strong $\mathrm{C}-\mathrm{H} \cdots \pi$ and $\mathrm{C}-\mathrm{H} \cdots \mathrm{N}$ intermolecular interactions could also hinder the molecular motions (Fig. 2D); however, the torsion and packing situations were different. The small pyrazine-(3-carbazole) dihedral angle of 25-B3C $\left(16^{\circ}\right)$ can lead to slight face-to-face stacking with a distance of ca. $3.3 \AA$ (Fig. 2C and F), which probably would not turn on its AIE activity. Because of the planar structure and no serious $\pi-\pi$ stacking, relatively high fluorescence efficiency in the solid state might be expected in 25-B3C.

\section{Photophysical properties}

The photophysical properties of these five compounds were analyzed based on the absorption spectra and photoluminescence (PL) spectra. As shown in Fig. 3A, the molecule 3C exhibits strong absorption bands at $300-370 \mathrm{~nm}$ in a THF solution with a peak at $329 \mathrm{~nm}$, corresponding to the $\pi-\pi^{*}$ transition of the whole skeleton; however, the absorption peaks of 26-B3C, TP-3C and 25-B3C shift to 334, 347 and $349 \mathrm{~nm}$, respectively, because of their larger molecular conjugation. Interestingly, the molecular conjugation of 23-B3C was considered to be larger than that of $3 \mathrm{C}$ because of an additional phenyl group on 23-B3C compared to 3C; however, 23-B3C showed a slightly blue-shifted absorption profile compared with $3 \mathrm{C}$, which may result from the twisted structure induced by the ortho-substituted phenyl group. Similarly, the absorption 


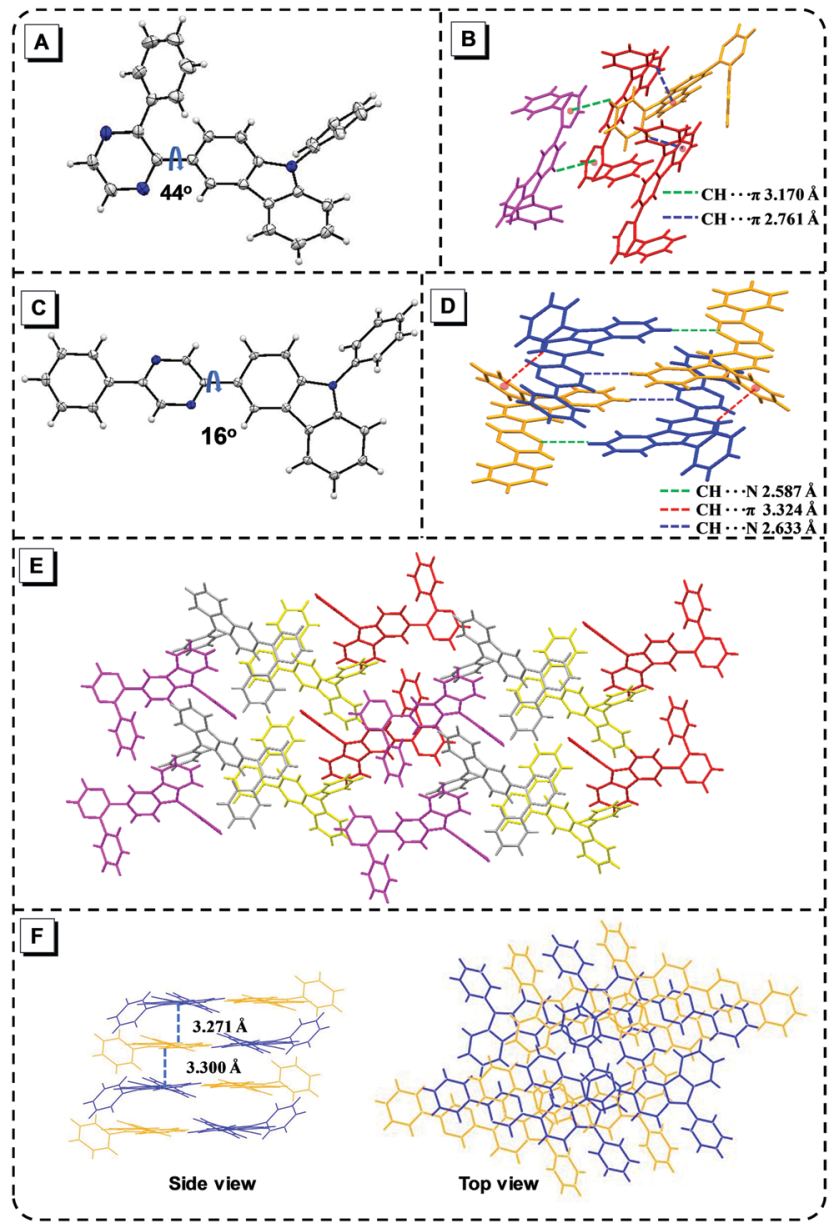

Fig. 2 Molecular structure in crystals of 23-B3C (A) and 25-B3C (C); intermolecular interactions in 23-B3C (B) and 25-B3C (D); crystal packing of 23-B3C (E) and 25-B3C (F).

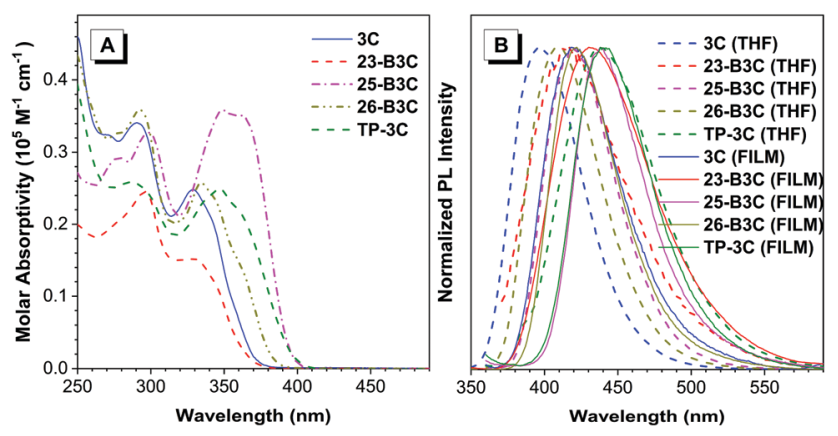

Fig. 3 (A) The absorption spectra of five compounds in THF solutions: (B) the PL spectra of five compounds in THF solutions and films.

peak of TP-3C (347 nm) was smaller than that of 25-B3C (349 nm) possibly because of its twisted structure. The series of pyrazine-based materials emitted violet and deep-blue fluorescence in both THF solutions and film states (Fig. 3B). For the 3-position substituted derivatives, 23-B3C and TP-3C showed weak emissions with fluorescence efficiency $(\Phi)$ values of 0.005 and 0.010 in THF solutions, while their $\Phi$ values were enhanced to 0.053 and 0.319 in film states, respectively (Table 1). For non-ortho-substituted compounds, enhancements in $\Phi$ were not observed with the states changing from solutions to films: $0.330,0.793$ and 0.396 in THF solutions and $0.134,0.694$ and 0.176 in film states for 3C, 25-B3C and 26-B3C, respectively; this indicated that the ortho-substituted compounds 23-B3C and TP-3C possessed AIE features, but substituting at either meta or para position did not endow 26-B3C and 25-B3C with classic AIE properties similar to the undecorated molecule 3C. Larger full width at half maximum (FWHM) values were observed for 23-B3C (75 nm) and TP-3C (76 nm) compared to other non-AIE active compounds ( $c a .55 \mathrm{~nm}$ ), which might indicate the flexibility of AIEgens. For meta-substituted derivatives, 26-B3C emitted slightly red-shifted fluorescence at $408 \mathrm{~nm}(0.396)$ and $421 \mathrm{~nm}(0.176)$ in THF solution and film state, respectively, while the PL spectra of the para-substituted 25-B3C were on the redder region with the emission peaks at $419 \mathrm{~nm}(0.793)$ in solution and $439 \mathrm{~nm}(0.694)$ in the film state. These results suggest that pyrazine compounds can be effectively tuned to obtain suitable fluorescence efficiency via inserting fluorophores at their meta positions; moreover, intense fluorescence can be observed with modifications at the para positions. For the triphenyl substituted situation, therefore, TP-3C integrates the PL performance from 23-B3C, 25-B3C and 26-B3C.

Their AIE nature was further validated through the investigation of the emission behaviors of the five compounds in $\mathrm{THF} /$ water mixtures (Fig. S16, ESI $\dagger$ ). For 23-B3C, its PL intensity gradually decreased with the emission peak shifting to the long wavelength region until the water fraction $\left(f_{\mathrm{w}}\right.$, vol\%) increased to $60 \%$ probably because of charge transfer (CT) emission. ${ }^{37}$ After this, it dramatically increased as the unconstrained molecules transformed to nanoaggregates. For TP-3C, its PL intensity slowly increased with the increase in the water fraction but remained low when $f_{\mathrm{w}}$ was less than $60 \%$, while it sharply increased when $f_{\mathrm{w}}$ was more than $60 \%$. The bright emission in nanoaggregates is attributed to the obstruction of non-radiative decay channels by restricted intramolecular motions. ${ }^{19}$ For non-AIE active compounds, 3C, 25-B3C and 26-B3C showed weaker fluorescence with the increase in $f_{\mathrm{w}}$ compared to pure THF, and this elucidated that meta and para substitution could not furnish AIE character again.

\section{Theoretical calculations}

As mentioned above, we speculate that substitution at three positions can achieve two effects; one is that bonding at the ortho-position can provide materials with AIE characteristics; the other is that insertion at the meta- or para-position can tune the emission wavelength and enhance fluorescence efficiency but cannot obtain AIE features. Herein, we chose 23-B3C and 25-B3C to discuss the AIE activity theoretically. The THF solution phase and aggregation effects were taken into account through the polarizable continuum model (PCM) and the combined quantum mechanics/molecular mechanics (QM/MM) approach, as implemented in the Gaussian 16 package. The geometrical structures were optimized by (time dependent) theoretical density functional ((TD)DFT) method with PBE0/6-31G(d) level. The natural 
Table 1 The photophysical properties of five compounds

\begin{tabular}{|c|c|c|c|c|c|c|c|c|c|c|c|c|c|}
\hline \multirow[b]{2}{*}{ Compound } & \multirow[b]{2}{*}{$\lambda_{\mathrm{abs}}{ }^{a}(\mathrm{~nm})$} & \multicolumn{4}{|c|}{$\underline{\lambda}^{b}{ }^{b}(\mathrm{~nm})$} & \multicolumn{2}{|l|}{$\Phi^{c}$} & \multicolumn{2}{|c|}{$\tau^{d}(\mathrm{~ns})$} & \multicolumn{2}{|c|}{$k_{\mathrm{r}}^{e}\left(10^{7} \mathrm{~s}^{-1}\right)$} & \multicolumn{2}{|c|}{$k_{\mathrm{nr}}^{f}\left(10^{7} \mathrm{~s}^{-1}\right)$} \\
\hline & & Soln & FWHM & Film & FWHM & Soln & Film & Soln & Film & Soln & Film & Soln & Film \\
\hline $3 \mathrm{C}$ & 329 & 397 & 55 & 420 & 52 & 0.330 & 0.134 & 1.70 & 2.29 & 19.4 & 5.9 & 39.4 & 37.8 \\
\hline 23-B3C & 328 & 415 & 75 & 430 & 78 & 0.005 & 0.053 & 0.96 & 1.37 & 0.5 & 3.9 & 103.6 & 69.1 \\
\hline $25-B 3 C$ & $349 / 363$ & 419 & 57 & 439 & 55 & 0.793 & 0.694 & 1.86 & 1.46 & 42.6 & 47.5 & 11.1 & 21.0 \\
\hline 26-B3C & 334 & 408 & 56 & 421 & 54 & 0.396 & 0.176 & 2.42 & 1.95 & 16.4 & 9.0 & 25.0 & 42.3 \\
\hline TP-3C & 347 & 437 & 76 & 440 & 61 & 0.010 & 0.319 & 0.88 & 0.71 & 1.1 & 44.9 & 112.5 & 95.9 \\
\hline
\end{tabular}

${ }^{a}$ Maximum absorption wavelength, concentration: $10^{-5}$ M. ${ }^{b}$ Maximum emission wavelength, soln: THF solution, film: neat film, FWHM: full width at half maximum. ${ }^{c}$ Absolute fluorescence quantum efficiency. ${ }^{d}$ Fluorescence lifetime. ${ }^{e} k_{\mathrm{r}}=\Phi / \tau .{ }^{f} k_{\mathrm{nr}}=(1-\Phi) / \tau$.

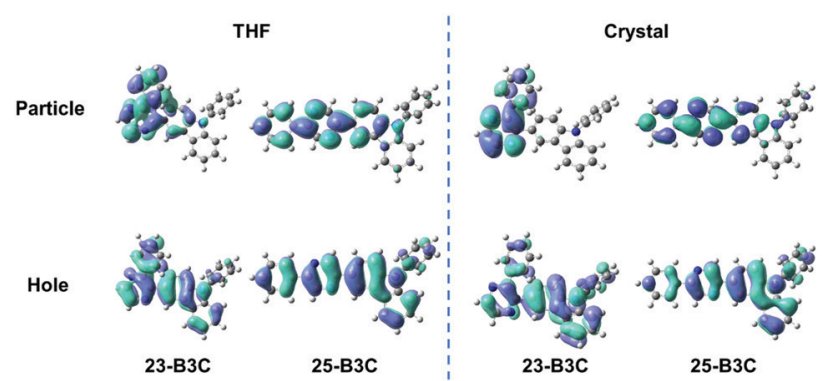

Fig. 4 The NTOs of 23-B3C and 25-B3C in THF solution and crystals.

transition orbitals (NTOs) were calculated to understand their electronic structure and all transition ratios from holes to particles were up to $99 \%$. As displayed in Fig. 4, the holes of 23-B3C and 25-B3C are dispersed on the whole molecules, while their particles are mainly determined by pyrazine and conjoint phenyl groups in both THF solutions and crystal states. In addition, 23-B3C showed more obvious orbital separation in crystals than in THF solutions, which indicated more CT proportion in crystals as expected and resulted in faint fluorescence. For 25-B3C, no remarkable disparity of NTO separation could be distinguished between solution and crystal states; thus, it can effectively fluoresce in both solution and solid states.

The reorganization energies of 23-B3C and 25-B3C in both solution and crystal states were investigated. The low-frequency $\left(\mathrm{LF},<200 \mathrm{~cm}^{-1}\right.$ ) and high-frequency (HF, 1400-1800 $\mathrm{cm}^{-1}$ ) modes and the related contributions to the total reorganization energies were obtained by normal mode analyses (Fig. 5 and Table S1, ESI $\dagger$ ). The LF modes mainly originated from the alteration of the dihedral angle by rotating phenyl and 3-carbazole groups, which could be hindered by molecular packing similar to many AIE compounds. The HF parts show the vibrations of intramolecular bonds and show little dependence on the stacking patterns. ${ }^{34,38-40}$ In solution, the total reorganization energy of $23-\mathrm{B} 3 \mathrm{C}$ is evaluated to be $529 \mathrm{meV}$ with $41 \%$ LF modes and $29 \%$ HF modes, indicating that many molecular rotations exist. In the crystal state, its total reorganization energy reduces to $329 \mathrm{meV}$ and the LF part decreases to $46 \mathrm{meV}$ from $218 \mathrm{meV}$. The large depression of the LF modes from solution to crystal states contributes to this AIE characteristic. Compared with the distinct reorganization energy change of 23-B3C, 25-B3C displays only $5 \mathrm{meV}$ difference on the LF modes between THF solution and crystal and these modes are not more than a quarter of the total reorganization energy; thus, no AIE phenomenon for 25-B3C is rational.

The changes in the dihedral angles of 23-B3C and 25-B3C from ground states to excited states were studied and listed in Table 2. In THF solution, 23-B3C shows a large conformation adjustment on excitation, e.g., $19.90^{\circ}$ of $\Delta\left(\alpha_{1-2-3-4}\right)$ but not for 25-B3C $\left(0.86^{\circ}\right.$ of $\left.\Delta\left(\alpha_{1-2-3-4}\right)\right)$. The difference between 23-B3C and 25-B3C implies that ortho-insertion can break pyrazine's planarity, reduce the rigidity and cause massive molecular motions. In crystals, all the changes in $\alpha$ decrease substantially, which demonstrates that the molecular rotations and pyrazine distortion are restrained, contributing to their AIE features.

Due to the lack of single crystal structures of 3C, 26-B3C and $\mathrm{TP}-3 \mathrm{C}$, their electronic structures and reorganization energies were computed only in the THF solution phase. Both $3 \mathrm{C}$ and 26-B3C showed relatively separated NTOs, while the local excited state occurred for TP-3C (Fig. S17, ESI $\dagger$ ). As illustrated in Fig. S18 (ESI $\dagger$ ), the total reorganization energies of $3 \mathrm{C}$ and 26-B3C are just 219 and $240 \mathrm{meV}$, and the LF parts are only 35 and $48 \mathrm{meV}$, respectively, less than $20 \%$ of the total value, respectively. Additionally, their pyrazine cores are only slightly warped (Table S2, ESI $\dagger$ ). Therefore, this implies that the good planarity of pyrazine also exists in 3C and 26-B3C and produces a little reorganization energy, which is advantageous for luminescence, similar to 25-B3C. However, in contrast to 25-B3C, an obvious fluorescence quenching effect was observed for $3 \mathrm{C}$ and 26-B3C probably because of the different stacking patterns that we could not investigate. On the contrary, TP-3C exhibited a considerably more reorganization energy of $406 \mathrm{meV}$ and its LF section of $120 \mathrm{meV}$ was larger than that of non-AIE molecules. The clear pyrazine's torsion was recorded in both $S_{0}$ and $S_{1}$ states, and it became more twisted after excitation (Table S2, ESI $\dagger$ ); this indicated the undermining of the pyrazine's planarity because steric hinderance caused considerable energy waste for molecular motions, similar 23-B3C. In the solid state, the restriction of these motions led to the AIE phenomenon. Two more phenyl groups modified on 23-B3C could increase its conjugation and rigidity, which explained the smaller reorganization energy of TP-3C than that of 23-B3C; therefore, TP-3C could emit more efficiently than 23-B3C in both solution and solid states.

\section{Demonstration of pyrazine's planarity and AIE}

To validate this point, we changed the chromophore and synthesized three ortho-disubstituted pyrazine compounds, 

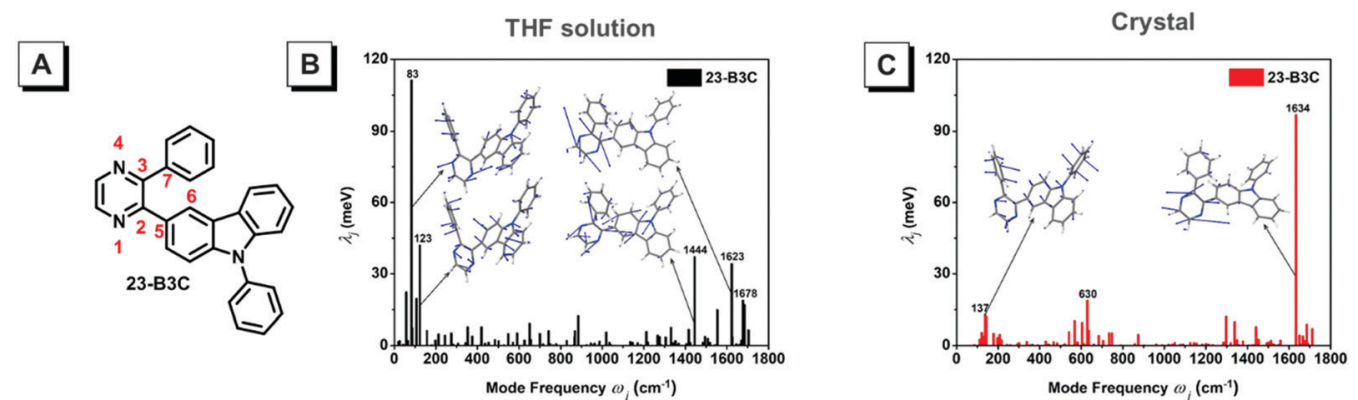

LF: $218 \mathrm{meV}, 41 \%$; HF: $155 \mathrm{meV}, 29 \%$; Total: $529 \mathrm{meV}$
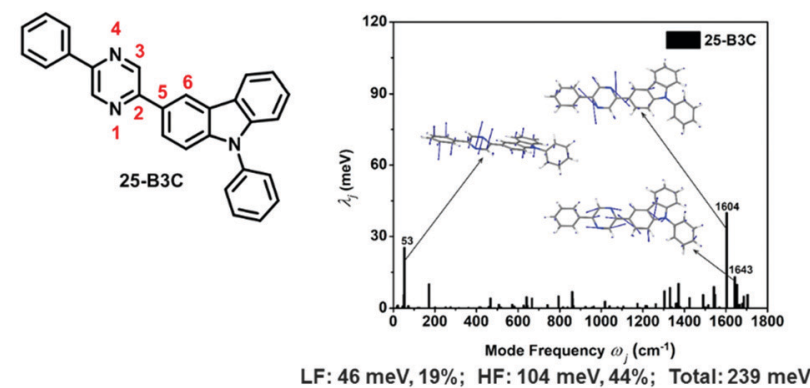

LF: $46 \mathrm{meV}, 14 \%$; HF: $145 \mathrm{meV}, 44 \%$; Total: $329 \mathrm{meV}$

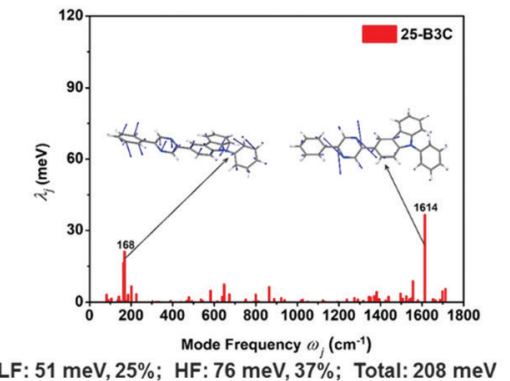

Fig. 5 (A) Atom label of 23-B3C and 25-B3C; the projection of the reorganization energy onto mode relaxations for two compounds (B) in THF solution and $(C)$ crystals.

Table 2 The selected dihedral angles of $23-B 3 C$ and $25-B 3 C$ in THF solutions and crystals

\begin{tabular}{|c|c|c|c|c|c|c|}
\hline & & \multicolumn{3}{|l|}{ 23-B3C } & \multicolumn{2}{|l|}{ 25-B3C } \\
\hline & & $\alpha_{1-2-3-4}$ & $\alpha_{3-2-5-6}$ & $\alpha_{5-2-3-7}$ & $\alpha_{1-2-3-4}$ & $\alpha_{1-2-5-6}$ \\
\hline THF & $\begin{array}{l}\mathrm{S}_{0} \\
\mathrm{~S}_{1} \\
\Delta\end{array}$ & $\begin{array}{r}-8.88 \\
-28.78 \\
19.90\end{array}$ & $\begin{array}{l}-40.07 \\
-16.47 \\
-23.60\end{array}$ & $\begin{array}{r}-12.46 \\
-39.55 \\
27.09\end{array}$ & $\begin{array}{r}-0.39 \\
0.47 \\
0.86\end{array}$ & $\begin{array}{r}-17.74 \\
-0.04 \\
-17.70\end{array}$ \\
\hline Crystal & $\begin{array}{l}\mathrm{S}_{0} \\
\mathrm{~S}_{1} \\
\Delta\end{array}$ & $\begin{array}{r}-6.85 \\
-8.09 \\
1.24\end{array}$ & $\begin{array}{r}-43.06 \\
-40.21 \\
-2.85\end{array}$ & $\begin{array}{r}-9.81 \\
-14.50 \\
4.69\end{array}$ & $\begin{array}{r}-1.02 \\
-1.61 \\
0.59\end{array}$ & $\begin{array}{r}-15.08 \\
-10.68 \\
-4.40\end{array}$ \\
\hline
\end{tabular}

namely, P-2PF, HP-2PF and PP-2PF for the flexibility of the mechanism (Fig. S19-S27, ESI $\dagger$ ). PP-2PF is the respective benzo-derivative of $\mathrm{P}-2 \mathrm{PF}$, and $\mathrm{HP}-2 \mathrm{PF}$ is the non-conjugated derivative. Their geometries were computed first. As shown in Table S3 (ESI $\dagger$ ), the $\Delta \alpha_{1-2-3-4}$ value of PP-2PF $\left(3.32^{\circ}\right)$ is smaller than that of $\mathrm{P}-2 \mathrm{PF}\left(16.91^{\circ}\right)$; therefore, the pyrazine's planarity of $\mathrm{PP}-2 \mathrm{PF}$ is better than that of P-2PF. When pyrazine is transformed to benzopyrazine (also called quinoxaline), the electronwithdrawing feature will be strengthened; thus, the apparently separated orbital distribution was acquired (Fig. S28, ESI $\dagger$ ). For HP-2PF, the structural change and NTOs were almost identical to those of P-2PF, which suggested that the non-conjugated modification did not affect its planarity. If pyrazine's planarity can be destroyed in the ortho-substituted mode and create numerous motions, considerable excited state energy will be consumed in the non-radiative relaxation path. As expected, P-2PF and HP-2PF showed obvious AIE, where the corresponding $\Phi$ values in THF were 0.022 and 0.021 , which then increased to 0.186 and 0.157 in film states, respectively. PP-2PF with good planarity exhibited moderate AIE: 0.155 in THF and
0.419 in film state (Fig. S29, S30 and Table S4, ESI $\dagger$ ). Therefore, these results demonstrate that the destruction of pyrazine's planarity leads to the AIE phenomenon.

\section{Device characterization}

Because of the suitable photoluminescence efficiency of 25-B3C, 26-B3C and TP-3C, we evaluated their electroluminescence (EL) performance. Previously, their thermal stability and morphological stability were tested by thermogravimetric analysis (TGA) and differential scanning calorimetry (DSC), respectively (Fig. S31, ESI $\dagger$ ). We observed good thermal stability with temperatures of $5 \%$ weight loss $\left(T_{\mathrm{d}}\right)$ as high as 343,315 and $386{ }^{\circ} \mathrm{C}$ and good morphological stability with the glass transition temperatures $\left(T_{\mathrm{g}}\right)$ of 60,60 and $119{ }^{\circ} \mathrm{C}$ for $25-\mathrm{B} 3 \mathrm{C}$, 26-B3C and TP-3C, respectively. Besides, the HOMO levels were determined from the onset of oxidation potential with the following equation: $E_{\mathrm{HOMO}}=-\left(E_{\text {onset }}-E_{\mathrm{Fc} / \mathrm{Fc}^{+}}+4.8\right) \mathrm{eV}$. The values of LUMOs were calculated using the following equation: $E_{\mathrm{HOMO}}=E_{\mathrm{LUMO}}+E_{\text {gap }}, E_{\text {gap }}=1240 / \lambda_{\text {onset }}$; here, $E_{\mathrm{Fc} / \mathrm{Fc}^{+}}$was $0.40 \mathrm{eV}$ and $\lambda_{\text {onset }}$ was calculated based on the onset of absorption in dichloromethane (Fig. S32, ESI $\dagger$ ). As shown in Table S2 (ESI $\dagger$ ), the HOMO values of 25-B3C, 26-B3C and TP-3C were estimated to be $-5.59,-5.54$, and $-5.55 \mathrm{eV}$, respectively. Their corresponding LUMO energy levels were then calculated to be $-2.42,-2.29$, and $-2.40 \mathrm{eV}$. It is feasible to apply them for blue emitters (Table S5, ESI $\dagger$ ).

Non-doped and 20 wt\%-doped OLED devices employing three materials as emitters with the configuration ITO/HATCN (5 nm)/TAPC (50 nm)/TCTA $(5 \mathrm{~nm}) /$ EML $(20 \mathrm{~nm}) /$ TmPyPB $(40 \mathrm{~nm}) / \mathrm{LiF}(1 \mathrm{~nm}) / \mathrm{Al}(120 \mathrm{~nm})$ were fabricated. In these devices, HATCN ((2,3,6,7,10,11-hexacyano-1,4,5,8,9,12-hexaazatriphe-nylene)) and TAPC (1,1-bis(4-di-p-tolylaminophenyl)cyclohexane) served 
Table 3 EL performance of OLED devices

\begin{tabular}{|c|c|c|c|c|c|c|c|}
\hline EML & $\lambda_{\mathrm{EL}}(\mathrm{nm})$ & $V_{\text {on }}^{b}(\mathrm{~V})$ & $L^{a}\left(\mathrm{~cd} \mathrm{~m}^{-2}\right)$ & $\eta_{\mathrm{c}}^{a}\left(\mathrm{~cd} \mathrm{~A}^{-1}\right)$ & $\eta_{\mathrm{P}}^{a}\left(\operatorname{lm~W^{-1})}\right.$ & $\mathrm{EQE}^{a}(\%)$ & $\operatorname{CIE}_{(x, y)}{ }^{c}$ \\
\hline 26-B3C & 420 & 2.9 & 1621 & 1.81 & 1.89 & 3.32 & $(0.16,0.07)$ \\
\hline TP-3C & 452 & 3.3 & 1672 & 1.97 & 1.73 & 1.54 & $(0.19,0.18)$ \\
\hline CBP:20 wt\% 26-B3C & 412 & 2.9 & 1007 & 0.78 & 0.76 & 2.85 & $(0.16,0.04)$ \\
\hline CBP:20 wt\% TP-3C & 444 & 3.1 & 1907 & 2.15 & 1.99 & 2.69 & $(0.15,0.10)$ \\
\hline
\end{tabular}

${ }^{a}$ The luminescence $(L)$, current efficiency $\left(\eta_{\mathrm{c}}\right)$, power efficiency $\left(\eta_{\mathrm{P}}\right)$ and external quantum efficiency are the maximum values of the devices. ${ }^{b} V_{\mathrm{on}}$ is the turn-on voltage at $1 \mathrm{~cd} \mathrm{~m}^{-2} .{ }^{c} \mathrm{CIE}$ coordinates at $10 \mathrm{~mA} \mathrm{~cm}{ }^{-2}$.

as the hole-injecting layer and hole-transporting layer, respectively. TCTA $\left(4,4^{\prime}, 4^{\prime \prime}\right.$-tri-9-carbazolytriphenylamine $)$ acted as the hole-transporting layer and electron-blocking layer, and TmPyPB (1,3,5-tri[(3-pyridyl)-phen-3-yl]benezene) functioned as both the electron-transporting layer and hole-blocking layer. CBP (4,4'-bis(carbazol-9-yl)biphenyl) was chosen as the host material.

As shown in Table 3 and Fig. 6A, these devices were turned on at a low voltage of 2.9-3.3 V, which was attributed to the excellent balance of carrier transport, and exhibited deep-blue to sky-blue electroluminescence emissions with peaks $\left(\lambda_{\mathrm{EL}}\right)$ from 412 to $452 \mathrm{~nm}$. Both doped and non-doped devices of 26-B3C showed the bluest fluorescence in this work. Among the non-doped OLED devices, 26-B3C performed the best with $L_{\max }$ of $1621 \mathrm{~cd} \mathrm{~m}^{-2}, \eta_{\text {ext,max }}$ of $3.32 \%$ and $\eta_{\mathrm{P}, \max }$ of $1.89 \mathrm{~lm} \mathrm{~W}^{-1}$ and deep-blue emission with CIE coordinates of $(0.16,0.07)$. Generally, doped blue OLED devices often display a blueshifted EL spectrum compared to non-doped ones; therefore, the EL spectrum of the doped devices shifted to 424, 412 and $444 \mathrm{~nm}$ and the values of $\mathrm{CIE}_{y}$ decreased to $0.06,0.04$ and 0.10 for 25-B3C, 26-B3C and TP-3C, respectively. In this work, the
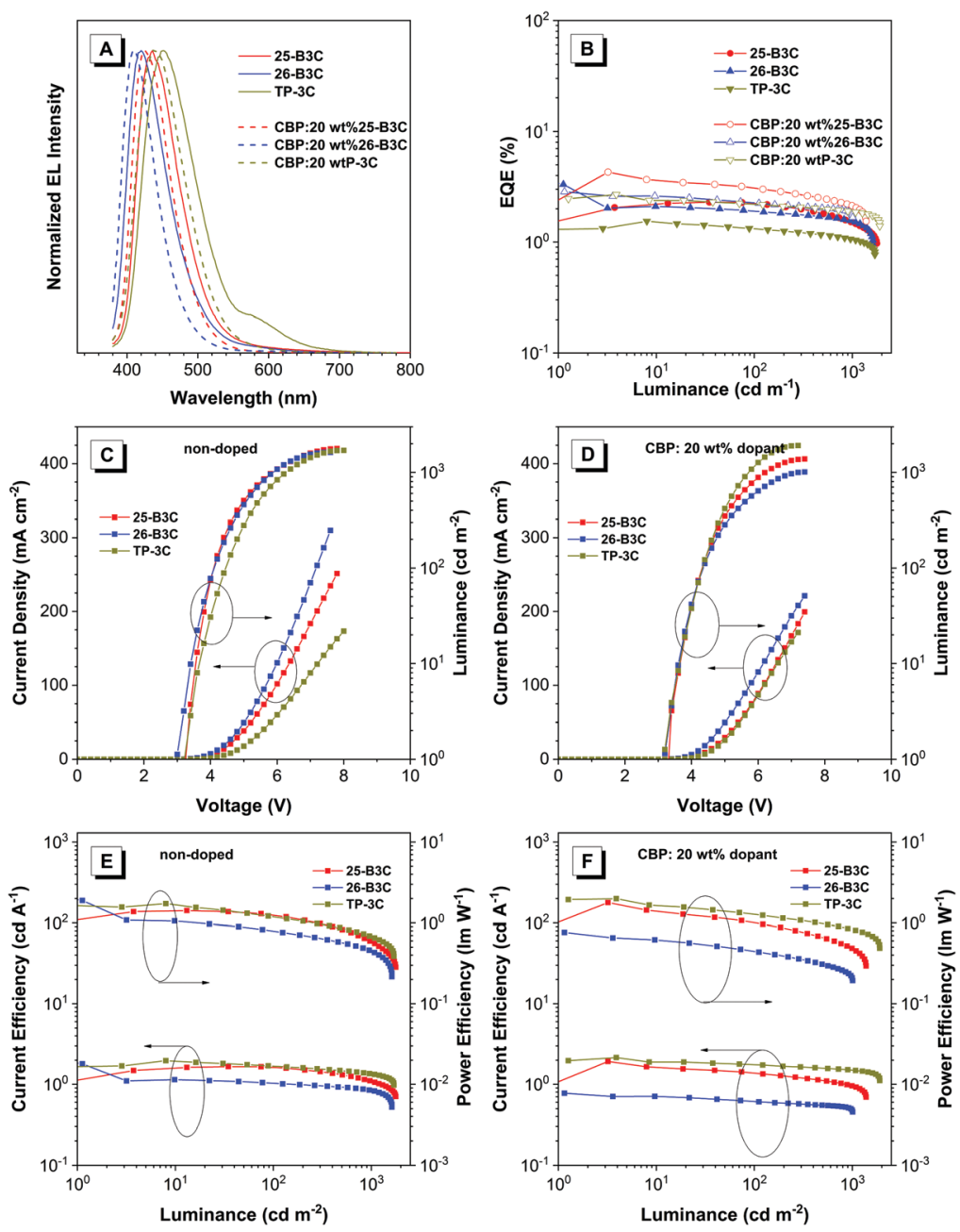

Fig. 6 The EL spectra, EQE-luminance, current density-voltage-luminance $(J-V-L)$ and current efficiency-luminance-power efficiency curves for these non-doped and doped devices. 
device of doped CBP:20 wt\% 25-B3C showed the best EL efficiency: $L_{\max }$ of $1383 \mathrm{~cd} \mathrm{~m}^{-2}, \eta_{\mathrm{P}, \max }$ of $1.78 \mathrm{~lm} \mathrm{~W}^{-1}, \eta_{\text {ext,max }}$ of $4.29 \%$ and CIE coordinates of (0.16 0.06).

\section{Conclusions}

Blue pyrazine fluorophores with three different bonding positions (ortho, meta and para) were synthesized and the substituted effect was investigated. A phenyl group inserted on the para site (25-B3C) could increase molecular conjugation and generate strong emission with red-shifted spectra; however, meta substitution (26-B3C) could improve its PL efficiency and tune its fluorescence, slightly. Different from the substitution at meta and para positions, substitution at the ortho position (23-B3C) could provide pyrazine-carbazole derivatives with AIE features because the steric hindrance of the ortho-phenyl unit disturbed pyrazine's planarity and switched on the non-radiative process, which has been verified in other pyrazine and benzopyrazine derivatives. For highly effective luminescence, the doped OLED device based on CBP:20 wt\% 25-B3C exhibited excellent deep-blue EL efficiency with an EQE of $4.29 \%$ and CIE coordinates of $(0.16,0.06)$. Therefore, high-efficiency luminescent materials can be exploited by this profound design.

\section{Conflicts of interest}

There are no conflicts to declare.

\section{Acknowledgements}

This work is financially supported by National Natural Science Foundation of China (21788102, 51673118, 21975077 and 21703122), Science \& Technology Program of Guangzhou (201804010218 and 201804020027), the Innovation and Technology Commission of Hong Kong (ITC-CNERC14S01), the Fundamental Research Funds for the Central Universities (D2190960), Fund of Key Laboratory of Luminescence from Molecular Aggregates of Guangdong Province (2019B030301003) and the Natural Science Foundation of Shandong Province (ZR2017BB034).

\section{Notes and references}

1 T. M. Figueira-Duarte and K. Müllen, Pyrene-based materials for organic electronics, Chem. Rev., 2011, 111, 7260.

2 M. Zhu and C. Yang, Blue fluorescent emitters: design tactics and applications in organic light-emitting diodes, Chem. Soc. Rev., 2013, 42, 4963.

3 Y. Tao, K. Yuan, T. Chen, P. Xu, H. Li, R. Chen, C. Zheng, L. Zhang and W. Huang, Thermally activated delayed fluorescence materials towards the breakthrough of organoelectronics, Adv. Mater., 2014, 26, 7931.

4 R. Ieuji, K. Goushi and C. Adachi, Triplet-triplet upconversion enhanced by spin-orbit coupling in organic light-emitting diodes, Nat. Commun., 2019, 10, 5283.
5 Y. Kondo, K. Yoshiura, S. Kitera, H. Nishi, S. Oda, H. Gotoh, Y. Sasada, M. Yanai and T. Hatakeyama, Narrowband deepblue organic light-emitting diode featuring an organoboronbased emitter, Nat. Photonics, 2019, 13, 678.

6 A. Zampetti, A. Minotto and F. Cacialli, Near-infrared (NIR) organic light-emitting diodes (OLEDs): challenges and opportunities, Adv. Funct. Mater., 2019, 29, 1807623.

7 J. Y. Zheng, Y. Yan, X. Wang, W. Shi, H. Ma, Y. S. Zhao and J. Yao, Hydrogen peroxide vapor sensing with organic core/sheath nanowire optical waveguides, Adv. Mater., 2012, 24, OP194.

8 M. Denis, J. Pancholi, K. Jobe, M. Watkinson and S. M. Goldup, Chelating rotaxane ligands as fluorescent sensors for metal ions, Angew. Chem., Int. Ed., 2018, 57, 5310.

9 L. Zhang, X. A. Liu, K. D. Gillis and T. E. Glass, A highaffinity fluorescent sensor for catecholamine: application to monitoring norepinephrine exocytosis, Angew. Chem., Int. Ed., 2019, 58, 7611.

10 R. Kumar, A. Sharma, H. Singh, P. Suating, H. S. Kim, K. Sunwoo, I. Shim, B. C. Gibb and J. S. Kim, Revisiting fluorescent calixarenes: from molecular sensors to smart materials, Chem. Rev., 2019, 119, 9657.

11 S.-N. Lei, H. Xiao, Y. Zeng, C.-H. Tung, L.-Z. Wu and H. Cong, BowtieArene: a dual macrocycle exhibiting stimuliresponsive fluorescence, Angew. Chem., Int. Ed., 2019, 59, 2.

12 B. A. D. Neto, P. H. P. R. Carvalho and J. R. Correa, Benzothiadiazole derivatives as fluorescence imaging probes: beyond classical scaffolds, Acc. Chem. Res., 2015, 48, 1560.

13 X. Xiong, F. Song, J. Wang, Y. Zhang, Y. Xue, L. Sun, N. Jiang, P. Gao, L. Tian and X. Peng, Thermally activated delayed fluorescence of fluorescein derivative for time-resolved and confocal fluorescence imaging, J. Am. Chem. Soc., 2014, 136, 9590.

14 A. Zhu, K. Miao, Y. Deng, H. Ke, H. He, T. Yang, M. Guo, Y. Li, Z. Guo, Y. Wang, X. Yang, Y. Zhao and H. Chen, Dually $\mathrm{pH} /$ reduction-responsive vesicles for ultrahigh-contrast fluorescence imaging and thermo-chemotherapy-synergized tumor ablation, ACS Nano, 2015, 9, 7874.

15 P. Agarwal, B. J. Beahm, P. Shieh and C. R. Bertozzi, Systemic fluorescence imaging of zebrafish glycans with bioorthogonal chemistry, Angew. Chem., Int. Ed., 2015, 54, 11504.

16 C. Yin, X. Zhen, H. Zhao, Y. Tang, Y. Ji, Y. Lyu, Q. Fan, W. Huang and K. Pu, Amphiphilic semiconducting oligomer for near-infrared photoacoustic and fluorescence imaging, ACS Appl. Mater. Interfaces, 2017, 9, 12332.

17 X. Wang, P. Li, Q. Ding, C. Wu, W. Zhang and B. Tang, Observation of acetylcholinesterase in stress-induced depression phenotypes by two-photon fluorescence imaging in the mouse brain, J. Am. Chem. Soc., 2019, 141, 2061.

18 G. v. Bünau, in Photophysics of aromatic molecules, ed. J. B. Birks, Wiley, London, 1970.

19 J. Mei, N. L. C. Leung, R. T. K. Kwok, J. W. Y. Lam and B. Z. Tang, Aggregation-induced emission: together we shine, united we soar!, Chem. Rev., 2015, 115, 11718.

20 S. Xu, Y. Duan and B. Liu, Precise molecular design for highperformance luminogens with aggregation-induced emission, Adv. Mater., 2020, 32, 1903530. 
21 R. Núñez, M. Tarrés, A. Ferrer-Ugalde, F. F. de Biani and F. Teixidor, Electrochemistry and photoluminescence of icosahedral carboranes, boranes, metallacarboranes, and their derivatives, Chem. Rev., 2016, 116, 14307.

22 A. V. Marsh, N. J. Cheetham, M. Little, M. Dyson, A. J. P. White, P. Beavis, C. N. Warriner, A. C. Swain, P. N. Stavrinou and M. Heeney, Carborane-induced excimer emission of severely twisted bis-o-carboranyl chrysene, Angew. Chem., Int. Ed., 2019, 57, 10640.

23 Y. Hong, J. W. Y. Lam and B. Z. Tang, Aggregation-induced emission: phenomenon, mechanism and applications, Chem. Commun., 2009, 4332.

24 Z. Liu, Z. Jiang, M. Yan and X. Wang, Recent progress of BODIPY dyes with aggregation-induced emission, Front. Chem., 2019, 7, 712.

25 X.-F. Zhang, Q. Xi and J. Zhao, Fluorescent and triplet state photoactive J-type phthalocyanine nano assemblies: controlled formation and photosensitizing properties, J. Mater. Chem., 2010, 20, 6726.

26 J. Shi, L. E. A. Suarez, S.-J. Yoon, S. Varghese, C. Serpa, S. Y. Park, L. Lüer, D. Roca-Sanjuán, B. Milián-Medina and J. Gierschner, Solid state luminescence enhancement in $\pi$-conjugated materials: unravelling the mechanism beyond the framework of AIE/AIEE, J. Phys. Chem. C, 2017, 121, 23166.

27 K. Kokado and K. Sada, Consideration of molecular structure in the excited state to design new luminogens with aggregationinduced emission, Angew. Chem., Int. Ed., 2019, 58, 8632.

28 C. Wang, Q. Qiao, W. Chi, J. Chen, W. Liu, D. Tan, S. McKechnie, D. Lyu, X.-F. Jiang, W. Zhou, N. Xu, Q. Zhang, Z. Xu and X. Liu, Quantitative design of bright fluorophores and AIEgens via the accurate prediction of twisted intramolecular charge transfer (TICT), Angew. Chem., Int. Ed., 2020, DOI: 10.1002/anie.201916357.

29 X. Liu, Q. Qiao, W. Tian, W. Liu, J. Chen, M. J. Lang and Z. Xu, Aziridinyl fluorophores demonstrate bright fluorescence and superior photostability by effectively inhibiting twisted intramolecular charge transfer, J. Am. Chem. Soc., 2016, 138, 6960.

30 Y. Luo, Y. Wang, S. Chen, N. Wang, Y. Qi, X. Zhang, M. Yang, Y. Huang, M. Li, J. Yu, D. Luo and Z. Lu, Facile access to twisted intramolecular charge-transfer fluorogens bearing highly pretwisted donor-acceptor systems together with readily fine-tuned charge-transfer characters, Small, 2017, 13, 1604113.
31 J. Li, C. Yang, X. Peng, Y. Chen, Q. Qi, X. Luo, W.-Y. Lai and W. Huang, Stimuli-responsive solid-state emission from $o$-carborane-tetraphenylethene dyad induced by twisted intramolecular charge transfer in the crystalline state, J. Mater. Chem. C, 2018, 6, 19.

32 Z. Zhao, X. Zheng, L. Du, Y. Xiong, W. He, X. Gao, C. Li, Y. Liu, B. Xu, J. Zhang, F. Song, Y. Yu, X. Zhao, Y. Cai, X. He, R. T. K. Kwok, J. W. Y. Lam, X. Huang, D. L. Phillips, H. Wang and B. Z. Tang, Non-aromatic annulene-based aggregation-induced emission system via aromaticity reversal process, Nat. Commun., 2019, 10, 2952.

33 M. Chen, L. Li, H. Nie, J. Tong, L. Yan, B. Xu, J. Z. Sun, W. Tian, Z. Zhao, A. Qin and B. Z. Tang, Tetraphenylpyrazine-based AIEgens: facile preparation and tunable light emission, Chem. Sci., 2015, 6, 1932.

34 L. Pan, H. Wu, J. Liu, K. Xue, W. Luo, P. Chen, Z. Wang, A. Qin and B. Z. Tang, Tetraphenylpyrazine based AIE luminogens: unique excited state decay and its application in deep-blue light-emitting diodes, Adv. Opt. Mater., 2019, 7, 1801673.

35 H. Wu, J. Zeng, Z. Xu, B. Zhang, H. Zhang, Y. Pan, Z. Wang, D. Ma, A. Qin and B. Z. Tang, Triphenylpyrazine: methyl substitution to achieve deep blue AIE emitters, J. Mater. Chem. C, 2019, 7, 13047.

36 Q. Li and Z. Li, The strong light-emission materials in the aggregated state: what happens from a single molecule to the collective group, Adv. Sci., 2017, 4, 1600484.

37 J. Mei, Y. Hong, J. W. Y. Lam, A. Qin, Y. Tang and B. Z. Tang, Aggregation-induced emission: the whole is more brilliant than the parts, Adv. Mater., 2014, 26, 5429.

38 X. Zheng, Q. Peng, L. Zhu, Y. Xie, X. Huang and Z. Shuai, Unraveling the aggregation effect on amorphous phase AIE luminogens: a computational study, Nanoscale, 2016, 8, 15173.

39 M. Chen, X. Hu, J. Liu, B. Li, N. L. C. Leung, L. Viglianti, T. S. Cheung, H. H. Y. Sung, R. T. K. Kwok, I. D. Williams, A. Qin, J. W. Y. Lam and B. Z. Tang, Rational design of red AIEgens with a new core structure from non-emissive heteroaromatics, Chem. Sci., 2018, 9, 7829.

40 Y.-C. Duan, Y. Wu, J.-L. Jin, D.-M. Gu, Y. Geng, M. Zhang and Z.-M. Su, Influence of aggregation on the structure and fluorescent properties of a tetraphenylethylene derivative: a theoretical study, ChemPhysChem, 2017, 18, 755. 